\title{
Evaluation des paramètres physiques, chimiques et bactériologiques des eaux d'une lagune tropicale en période d'étiage : la lagune Aghien (Côte d'Ivoire)
}

\author{
Abou TRAORE ${ }^{1 *}$, Gbombélé SORO ${ }^{1}$, Emmanuel Konan KOUADIO ${ }^{1}$, \\ Barthélémy Siaka BAMBA ${ }^{2}$, Marie Solange OGA $^{1}$, Nagnin SORO ${ }^{1}$ et Jean BIEMI ${ }^{1,3}$ \\ ${ }^{1}$ Laboratoire des Sciences et Techniques de l'Eau et de l'Environnement, UFR des Sciences de la terre et des \\ ressources Minières 22 BP 582 Abidjan 22, Université Félix Houphouët Boigny de Cocody, \\ Abidjan, Côte D'Ivoire. \\ ${ }^{2}$ Centre de Recherches Océanologiques, BP V18 Abidjan, Côte D'Ivoire. \\ ${ }^{3}$ Centre Universitaire de Recherche et d'Application en Télédétection (CURAT), UFR des Sciences de la Terre \\ et des Ressources Minières, 22 BP 801 Abidjan 22, Côte D'Ivoire. \\ *Auteur correspondant, E-mail: bouareta@yahoo.fr ; aboutraoreat@gmail.com; Tél :(+225)07278348.
}

\section{RESUME}

Pour pallier le déficit en eau potable du District d'Abidjan, la lagune Aghien est pressentie pour venir en appoint aux champs de captages existants. La présente étude se propose d'améliorer les connaissances sur les qualités physiques, chimiques et bactériologiques des eaux de cette lagune en période d'étiage. Certains paramètres physiques et chimiques ont été mesurés in situ à l'aide d'appareils multifonctionnels et d'autres ont été analysées au laboratoire par des méthodes hydrochimiques homologuées par l'Agence Française de Normalisation (AFNOR). Cette étude montre que les eaux de la lagune Aghien, quasi exclusivement alimentée par les eaux continentales, sont basiques, avec un $\mathrm{pH}$ moyen de 7,6 qui dénote une activité biologique très intense dans la lagune. Elles ont une salinité moyenne de 0,03 et une conductivité électrique faible qui varie entre 80,6 et $198,7 \mu \mathrm{S} . \mathrm{cm}^{-1}$. Elles contiennent un faible taux d'oxygène dissous, avec une moyenne de $66,83 \%$ et elles sont riches en matières organiques. D'importantes valeurs de $\mathrm{DBO}_{5}$ y ont été déterminées, avec une moyenne de $17,46 \mathrm{mg} . \mathrm{L}^{-1}$. La lagune Aghien est un milieu réducteur, avec un potentiel d'oxydoréduction moyen de $-67,7 \mathrm{mV}$ et ses eaux sont confrontées à une pollution chimique, avec une teneur moyenne en ion ammonium de 23,04 mg.L. $\mathrm{L}^{-1}$ supérieure à la valeur guide de l'OMS $\left(0,05 \mathrm{mg} . \mathrm{L}^{-1}\right)$. Les eaux de cette lagune ont une mauvaise qualité bactériologique. Elles contiennent par endroit de fortes teneurs en coliformes fécaux (520 $\mathrm{UFC} / \mathrm{ml})$ et en coliformes totaux $(550 \mathrm{UFC} / \mathrm{ml})$ et elles contiennent également des streptocoques fécaux (2 $\mathrm{UFC} / \mathrm{ml})$.

() 2012 International Formulae Group. All rights reserved.

Mots clés: Lagune Aghien, paramètres physiques, paramètres chimiques, appareil multifonctionnels, qualité bactériologique.

\section{INTRODUCTION}

L'un des objectifs majeurs des projets de développement des états à travers le monde est l'accessibilité des populations à l'eau potable. Abidjan, la capitale économique de la 
Côte d'Ivoire connait une démographie galopante et une urbanisation accélérée qui creusent le fossé entre l'offre et la demande en eau potable (MEF, 2003). Abidjan et ses alentours sont alimentés par les eaux souterraines du Continental Terminal. La qualité de ces eaux est sans cesse dégradée par des polluants industriels et domestiques. Ainsi, la pollution nitratée signalée par Patrice et al. (2006) et Ernest et al. (2008) a occasionné l'abandon total de certains champs captants. Face à cette situation, les eaux de surface notamment celles de la lagune Aghien pourraient être utilisées pour l'approvisionnement des populations en eau potable. Les eaux de cette lagune ont un caractère fluvial et sont toute l'année quasi exclusivement d'origine continentale (JeanRené et Daniel, 1994). La lagune Aghien est un écosystème rural et donc susceptible d'être moins polluée. Cependant, sa proximité avec de nombreux villages, des plantations et des fermes d'élevage pourrait entraîner la dégradation de ses eaux. En effet, elle est utilisée pour la pêche, la production piscicole et pour les activités ménagères notamment la vaisselle et la lessive ou comme eau de baignade. Les rejets domestiques (eaux usées, ordures ménagères, fèces, etc.) des riverains sont déversés directement dans les eaux lagunaires. En outre, les eaux de lessivage des terres agricoles utilisant des produits phytosanitaires ruissellent vers la lagune. L'appréciation de la qualité des eaux de surface se base sur la mesure de paramètres physico-chimiques et chimiques ainsi que sur la présence ou l'absence d'organismes et de micro-organismes aquatiques, indicateurs d'une plus ou moins bonne qualité de l'eau (Juliette et al., 2005). La présente étude se propose donc d'améliorer les connaissances sur les qualités physiques, chimiques et bactériologiques des eaux de la lagune Aghien en vue de leur utilisation pour l'approvisionnement en eau potable.

\section{MATERIEL ET METHODES}

Zone d'étude

La zone d'étude est située au Sud-Est de la Côte d'Ivoire entre les latitudes $5^{\circ} 21^{\prime} \mathrm{N}$ et $5^{\circ} 28^{\prime} \mathrm{N}$ et les longitudes $3^{\circ} 49^{\prime} \mathrm{W}$ et $3^{\circ} 58^{\prime} \mathrm{W}$ (Figure 1). La lagune Aghien est située entre les latitudes $5^{\circ} 22^{\prime} \mathrm{N}$ et $5^{\circ} 26^{\prime} \mathrm{N}$ et les longitudes $3^{\circ} 49^{\prime} \mathrm{W}$ et $3^{\circ} 55^{\prime} \mathrm{W}$. Elle se trouve au Nord de la lagune Ebrié de laquelle elle est séparée par la lagune Potou. Les lagunes Aghien et Potou communiquent par un chenal naturel. Le climat de la zone d'étude est de type équatorial caractérisé par quatre saisons dont une grande saison sèche de décembre à mars, une grande saison de pluie d'avril à juillet, une petite saison sèche d'août à septembre et une petite saison de pluie d'octobre à novembre. C'est une zone pluvieuse, avec des précipitations interannuelles supérieures à $1500 \mathrm{~mm}$. La région est essentiellement couverte par la forêt dense sempervirente et ombrophile fortement dégradée à cause des activités anthropiques en l'occurrence, l'expansion des cultures de rente (Palmier à huile, hévéas) et la croissance de l'habitat humain. La zone renferme un réseau hydrographique très important composé des rivières Bété, Djibi et Mé. La Bété et la Djibi débouchent directement dans la lagune Aghien et la Mé débouche dans le canal naturel entre les lagunes Aghien et Potou.

La géologie de la zone d'étude est marquée par deux unités géologiques dont les formations sédimentaires situées au Sud et les formations du socle cristallin et cristallophyllien situées à l'extrême Nord. Les formations sédimentaires sont constituées de roches détritiques du Tertiaire et du Quaternaire. Le bassin sédimentaire, qui occupe la majeure partie de la zone, présente 
des sables et des vases quaternaires sur le bord Est de la lagune Aghien et le long de la rivière Mé. Les sables, argiles et grès ferrugineux du Continental Terminal sont les plus abondants. Les formations du socle cristallin et cristallophyllien sont constituées de gneiss fins à biotite et amphibole (Claude et al., 1992). Deux types d'aquifères sont rencontrés dans la zone d'Aghien en l'occurrence, les aquifères discontinus du socle et les aquifères continus du bassin sédimentaire.

\section{Echantillonnage et techniques d'analyse}

Les analyses ont été réalisées sur des échantillons d'eau prélevés dans la lagune Aghien en période d'étiage en mars 2010 dans dix stations bien réparties sur la lagune. Les analyses chimiques ont été effectuées sur vingt échantillons dont dix ont été prélevés à $20 \mathrm{~cm}$ de la surface et les 10 autres à $50 \mathrm{~cm}$ de l'interface eau/sédiment et l'analyse bactériologique a porté sur 10 échantillons. La température $\left(\mathrm{T}^{\circ} \mathrm{C}\right)$, le $\mathrm{pH}$, l'oxygène dissous (OD) et le potentiel redox (Eh) ont été mesurés in situ à l'aide d'un multiparamètre de type THERMO ELECTRON CORPORATION ORION 4 STAR aux précisions $\pm 0,1\left(0-70{ }^{\circ} \mathrm{C}\right) ; 0,002 ; 0,01$ et 0,2 respectivement. La conductivité électrique (CE), la salinité et les solides dissous totaux (TDS) ont été mesurés in situ à l'aide d'un autre appareil multifonctionnel de type $\mathrm{HACH}$ sension 5 aux précisions $\pm 0,005 ; 0,1$ et 0,005 respectivement. La transparence a été mesurée in situ à l'aide d'un disque de Secchi et la profondeur a été obtenue à partir de la corde graduée attachée à la bouteille de Niskin qui a servi pour le prélèvement des échantillons d'eau. Les échantillons d'eau destinés aux analyses de laboratoire ont été recueillis dans des bouteilles en plastique et en verre et transportés dans des glacières au Laboratoire Central pour l'Hygiène Alimentaire et l'Agroindustrie (LCHAI). Les matières en suspension y ont été analysées par la méthode par centrifugation (NF, 1978). La méthode par acidimétrie après distillation (NF, 1975) a été utilisée pour la mesure de l'ion ammonium à l'aide d'un distillateur de type MRK KJELDAHL-AUTO VAPOR-STILL USSA1. Le dosage des ions phosphates a été réalisé par la méthode de Murphy et Riley (1962) à l'aide d'un spectrophotomètre de type UV-1700 PHARMASPEC, UV-VIS SPECTROPHO-TOMETER à la précision $\pm 0,3$. La détermination de la concentration des nitrates et des nitrites a été effectuée selon la Norme Française (1987) à l'aide d'un spectrophotomètre d'absorption moléculaire UV/VIS-JASCO V-530 à la précision $\pm 0,3$. Les paramètres microbiologiques ont été déterminés par ensemencement des microbes dans des milieux de culture spécifiques à chaque type de bactérie. Ainsi, les milieux suivants ont été utilisés pour la recherche et le dénombrement des microbes: le milieu VRBL pour les coliformes totaux et fécaux, le milieu SLANETZ et BARTLEY pour les streptocoques fécaux, le milieu T.C.B.S. pour les vibrions et le milieu HEKTOEN pour les salmonelles. 


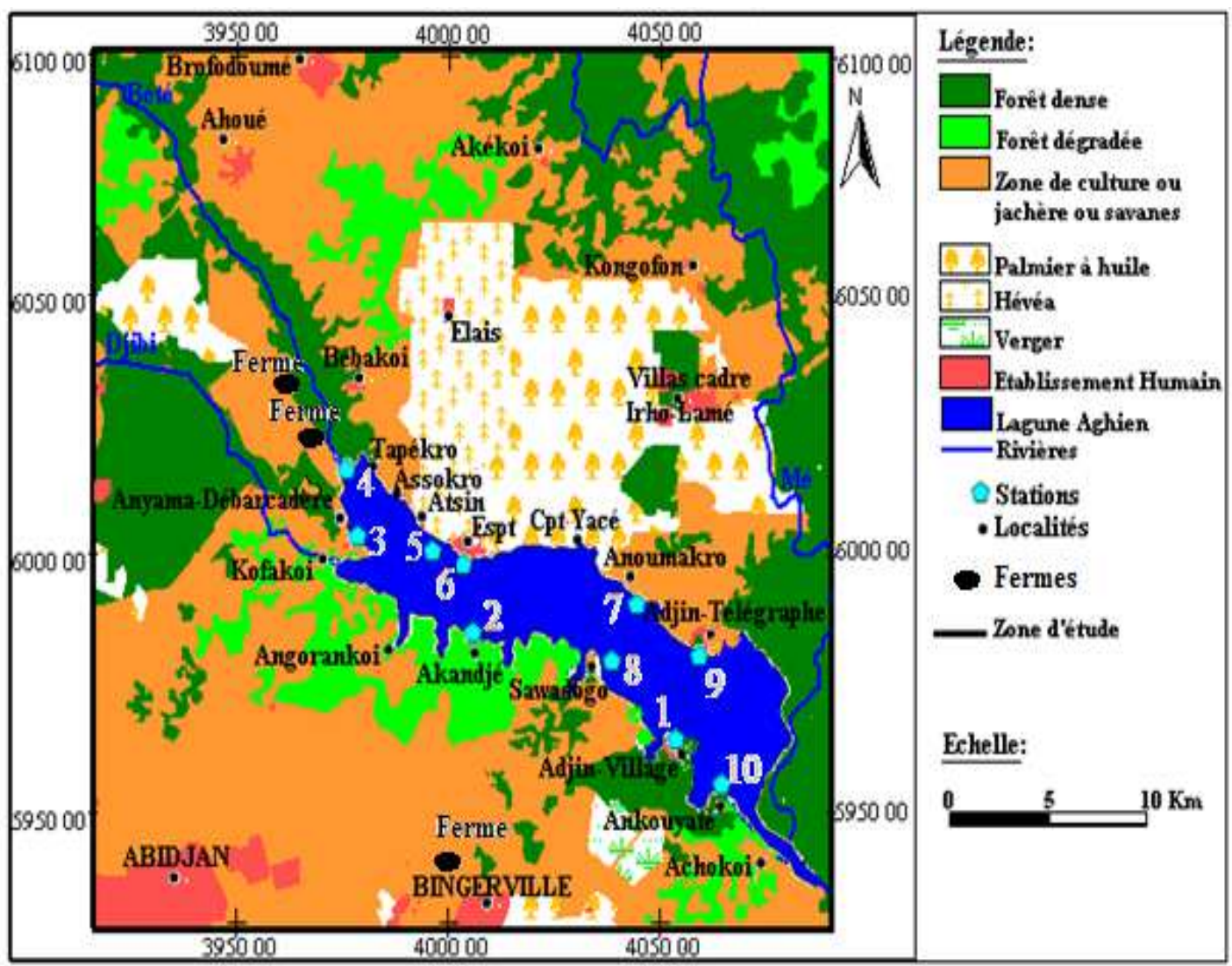

Figure 1: Occupation du sol et localisation des stations d'échantillonnage sur la lagune Aghien.

\section{RESULTATS}

Les résultats de l'analyse des paramètres physiques et chimiques réalisée sur les eaux de la lagune Aghien sont présentés dans le Tableau 1 et 2 respectivement. La température des eaux de la lagune Aghien varie entre $31,8{ }^{\circ} \mathrm{C}$ et $33{ }^{\circ} \mathrm{C}$, avec une moyenne de $32,3{ }^{\circ} \mathrm{C}$ et le $\mathrm{pH}$ moyen est de 7,6. Les eaux de la lagune Aghien sont oligohalines, la salinité varie de 0 à 0,1 , avec une moyenne de 0,03 . La conductivité électrique des eaux est faible et comprise entre 80,6 et $198,7 \mu{\mathrm{S} . \mathrm{cm}^{-1}}$, avec une moyenne de $110,0 \mu \mathrm{S} . \mathrm{cm}^{-1}$. Les teneurs en solides totaux dissous (TDS) vont de 38,1 à 95,0 mg..-1, avec une moyenne de 52,2 mg. $\mathrm{L}^{-1}$. Les concentrations de la lagune en oxygène dissous sont faibles, elles sont comprises entre
$3,60 \mathrm{mg} \cdot \mathrm{L}^{-1}(48,90 \%)$ et $6,70 \mathrm{mg} \cdot \mathrm{L}^{-1}$ $(91,25 \%)$, avec une moyenne de $4,90 \mathrm{mg} . \mathrm{L}^{-1}$ $(66,83 \%)$. $\mathrm{La}^{\mathrm{DBO}} 5$ a des valeurs comprises entre 10,95 et $26,63 \mathrm{mg} . \mathrm{L}^{-1}$, avec une moyenne de $17,46 \mathrm{mg} . \mathrm{L}^{-1}$. Les valeurs du potentiel redox sont comprises entre - 140,2 et - 8,3 mV, avec une moyenne de - 67,7 mV. Les teneurs des matières en suspension (MES) sont comprises entre 6,75 et $18,37 \mathrm{mg} . \mathrm{L}^{-1}$, avec une moyenne de $14,11 \mathrm{mg} . \mathrm{L}^{-1}$. La transparence varie entre 0,60 et $1,50 \mathrm{~m}$, avec une valeur moyenne de $0,96 \mathrm{~m}$.

La concentration en nitrates se situe entre 1,04 et 7,58 mg. $\mathrm{L}^{-1}$, avec une moyenne de 4,25 mg. $\mathrm{L}^{-1}$. Les concentrations en nitrites varient entre 0,002 et $0,006 \mathrm{mg} . \mathrm{L}^{-1}$, avec une moyenne de $0,004 \mathrm{mg} . \mathrm{L}^{-1}$. Les concentrations 
en nitrates et nitrites sont faibles par rapport aux normes OMS (2004) qui sont 50 et 0,1 mg. $\mathrm{L}^{-1}$ respectivement. Les teneurs de $\mathrm{NH}_{4}{ }^{+}$ varient entre 7,20 et 50,40 mg. $\mathrm{L}^{-1}$, avec une moyenne de 23,04 mg. $\mathrm{L}^{-1}$. Ces valeurs sont largement supérieures à la norme OMS qui est de $0,05 \mathrm{mg} . \mathrm{L}^{-1}$ pour les eaux destinées à la consommation humaine. Les concentrations de $\mathrm{PO}_{4}{ }^{3-}$ sont comprises entre 0 et $0,43 \mathrm{mg}$. $\mathrm{L}^{-}$ 1 , avec une moyenne de $0,05 \mathrm{mg} . \mathrm{L}^{-1}$.

Les résultats de l'analyse microbiologique sont présentés dans le Tableau 3. Les bactéries de genre salmonella et les virus de genre vibrio sont absentes des eaux de la lagune Aghien. Aucune colonie caractéristique des streptocoques fécaux n'est comptée (résultats <1) aux stations $3 ; 4 ; 5 ; 6 ; 7 ; 8$ et 10 . Par contre, elles sont présentes aux stations 1 , avec $1 \mathrm{UFC} / \mathrm{ml}$, à la station 2, avec $2 \mathrm{UFC} / \mathrm{ml}$ et à la station 9, avec $2 \mathrm{UFC} / \mathrm{ml}$. En comparant ces résultats à la norme OMS (1992) qui est 0 UFC/100ml. Il apparaît une légère pollution aux stations $1 ; 2$ et 9 . Pour les coliformes fécaux, aucune colonie caractéristique n'est comptée (résultats <1) aux stations $5 ; 7$ et 8 . Ces bactéries sont présentes aux stations 1 (70 UFC/ml); 2 (520 UFC/ml); 3 (140 UFC/ml); 4 et 6 , avec $10 \mathrm{UFC} / \mathrm{ml}$; aux stations 9 et 10 , avec respectivement 90 et $98 \mathrm{UFC} / \mathrm{ml}$. La norme OMS (1992) étant de $0 \mathrm{UFC} / 100 \mathrm{ml}$, ces résultats traduisent une pollution bactérienne. Les plus fortes valeurs sont observées aux stations 2 et 3 . Les coliformes totaux ne présentent aucune colonie caractéristique (résultats < 1) à la station 7 . Pour toutes les autres stations, ces bactéries sont présentes, avec des concentrations très souvent élevées comparativement à la norme OMS (1992) (10 $\mathrm{UFC} / \mathrm{ml}$ ). Ainsi on a $280 \mathrm{UFC} / \mathrm{ml}$ aux stations 1 et $9 ; 550 \mathrm{UFC} / \mathrm{ml}$ à la station $2 ; 350 \mathrm{UFC} / \mathrm{ml}$ à la station $3 ; 180 \mathrm{UFC} / \mathrm{ml}$ à la station $4 ; 10$ $\mathrm{UFC} / \mathrm{ml}$ aux stations 5,6 et 8 et $120 \mathrm{UFC} / \mathrm{ml}$ à la station 10 .

Tableau 1: Paramètres physiques des eaux de la lagune Aghien (Mars 2010).

\begin{tabular}{ccccc}
\hline & $\mathbf{T}^{\circ} \mathbf{C}$ & Salinité & MES $\left(\mathbf{m g}, \mathbf{L}^{-\mathbf{1}}\right)$ & Transparence $(\mathbf{m})$ \\
\hline station 1 & 32,45 & 0,1 & 16,38 & 0,8 \\
station 2 & 32,00 & 0 & 17,63 & 1,1 \\
station 3 & 32,40 & 0 & 18,38 & 0,95 \\
station 4 & 31,80 & 0 & 18,13 & 0,8 \\
station 5 & 32,10 & 0 & 11,00 & 1 \\
station 6 & 33,00 & 0 & 6,75 & 0,9 \\
station 7 & 32,60 & 0 & 7,63 & 0,95 \\
station 8 & 32,50 & 0 & 15,38 & 1 \\
station 9 & 31,75 & 0,1 & 15,75 & 1,5 \\
station 10 & 32,05 & 0,1 & & 0,6 \\
Moyenne & $\mathbf{3 2 , 2 7}$ & $\mathbf{0 , 0 3}$ & $\mathbf{1 4 , 1 1}$ & $\mathbf{0 , 9 6}$ \\
Ecarttype & $\mathbf{0 , 3 7}$ & $\mathbf{0 , 0 5}$ & $\mathbf{4 , 2 4}$ & $\mathbf{0 , 2 2}$ \\
Coef.Var & $\mathbf{1 , 1 5}$ & $\mathbf{1 5 2 , 7 5}$ & $\mathbf{3 0 , 0 6}$ & $\mathbf{2 3 , 2 7}$ \\
\hline
\end{tabular}

MES : teneurs des matières en suspension ; $\mathrm{T}^{\circ} \mathrm{C}$ : température 
Tableau 2: Paramètres chimiques des eaux de la lagune Aghien (Mars 2010).

\begin{tabular}{|c|c|c|c|c|c|}
\hline & pH & $\begin{array}{c}\text { OD } \\
\left(\mathrm{mg} \cdot \mathrm{L}^{-1}\right)\end{array}$ & $\begin{array}{c}\mathrm{CE} \\
\left(\mu \mathrm{S} . \mathrm{cm}^{-1}\right)\end{array}$ & $\begin{array}{c}\text { TDS } \\
\left(\mathrm{mg}^{\left.-L^{-1}\right)}\right.\end{array}$ & Eh $(\mathrm{mV})$ \\
\hline station 1 & 7,1 & 4,74 & 144,30 & 68,80 & $-39,85$ \\
\hline station 2 & 7,5 & 4,18 & 92,05 & 43,65 & $-62,30$ \\
\hline station 3 & 7,1 & 4,04 & 80,55 & 38,10 & $-39,90$ \\
\hline station 4 & 7,5 & 4,92 & 81,80 & 38,70 & $-59,60$ \\
\hline station 5 & 8,2 & 5,32 & 84,60 & 40,05 & $-104,35$ \\
\hline station 6 & 8,7 & 6,03 & 87,45 & 41,40 & $-131,15$ \\
\hline station 7 & 8,8 & 6,69 & 102,30 & 48,50 & $-140,20$ \\
\hline station 8 & 7,3 & 4,05 & 98,10 & 46,50 & $-47,15$ \\
\hline station 9 & 7,2 & 5,45 & 129,60 & 61,70 & $-44,45$ \\
\hline station 10 & 6,6 & 3,59 & 198,70 & 94,95 & $-8,30$ \\
\hline Moyenne & 7,6 & 4,90 & 109,95 & 52,24 & $-67,73$ \\
\hline Coef.Var & 9,01 & 19,00 & 32,50 & 32,93 & $-60,45$ \\
\hline \multirow[t]{2}{*}{ Ecarttype } & 0,68 & 0,93 & 35,73 & 17,20 & 40,94 \\
\hline & $\begin{array}{c}\mathrm{DBO}_{5} \\
\left(\mathrm{mg} \cdot \mathrm{L}^{-1}\right)\end{array}$ & $\begin{array}{c}\mathrm{NO}_{3}^{-} \\
\left(\mathrm{mg} . \mathrm{L}^{-1}\right)\end{array}$ & $\begin{array}{c}\mathrm{NO}_{2}^{-} \\
\left(\mathrm{mg} \cdot \mathrm{L}^{-1}\right)\end{array}$ & $\begin{array}{c}\mathrm{NH}_{4}^{+} \\
\left(\mathrm{mg} . \mathrm{L}^{-1}\right)\end{array}$ & $\begin{array}{c}\mathrm{PO}_{4}^{3-} \\
\left(\mathrm{mg} . \mathrm{L}^{-1}\right)\end{array}$ \\
\hline station 1 & 25,10 & 4,86 & 0,003 & 7,2 & 0,006 \\
\hline station 2 & 26,63 & 3,48 & 0,005 & 28,8 & 0,000 \\
\hline station 3 & 14,19 & 4,34 & 0,003 & 14,4 & 0,001 \\
\hline station 4 & 10,98 & 5,46 & 0,003 & 21,6 & 0,000 \\
\hline station 5 & 11,13 & 3,04 & 0,002 & 36 & 0,010 \\
\hline station 6 & 21,84 & 3,17 & 0,004 & 7,2 & 0,410 \\
\hline station 7 & 10,96 & 5,23 & 0,004 & 50,4 & 0,037 \\
\hline station 8 & 16,55 & 7,59 & 0,004 & 7,2 & 0,081 \\
\hline station 9 & 16,86 & 4,36 & 0,003 & 21,6 & 0,034 \\
\hline station 10 & 20,41 & 1,04 & 0,007 & 36 & 0,002 \\
\hline Moyenne & 17,46 & 4,26 & 0,004 & 23,04 & 0,058 \\
\hline Coef.Var & 31,71 & 38,94 & 29,75 & 60,60 & 206,37 \\
\hline Ecarttype & 5,54 & 1,66 & $\mathbf{0 , 0 0 1}$ & 13,96 & 0,12 \\
\hline
\end{tabular}

OD : Oxygène Dissous ; CE : Conductivité Electrique, TDS : Solides Dissous Totaux.

Tableau 3: Paramètres microbiologiques des eaux de la lagune Aghien (Mars 2010).

\begin{tabular}{lccccc}
\hline & $\begin{array}{c}\text { Coliformes } \\
\text { totaux } \\
(\mathbf{U F C} / \mathbf{m l}) \\
(\text { ISO 9308-1) }\end{array}$ & $\begin{array}{c}\text { Coliformes } \\
\text { fécaux } \\
(\mathbf{U F C} / \mathbf{m l})\end{array}$ & $\begin{array}{c}\text { Streptocoques } \\
\text { (ISO 930)-1) } \\
(\mathbf{U F C} / \mathbf{m l})\end{array}$ & $\begin{array}{c}\text { Salmonella } \\
(\mathbf{U S F C} / \mathbf{m l}) \\
(\mathbf{I S O})\end{array}$ & $\begin{array}{c}\text { Vibrion } \\
(\mathbf{U F C} / \mathbf{m l}) \\
(\mathbf{N F}\end{array}$ \\
\hline Station 1 & 280 & 70 & 01 & absent & absent \\
ISO6579) & ISO8914) \\
Station 2 & 550 & 520 & 02 & absent & absent \\
Station 3 & 350 & 140 & $<1$ & absent & absent \\
Station 4 & 180 & 10 & $<1$ & absent & absent
\end{tabular}




\begin{tabular}{lccccc} 
Station 5 & 10 & $<1$ & $<1$ & absent & absent \\
Station 6 & 10 & 10 & $<1$ & absent & absent \\
Station 7 & $<1$ & $<1$ & $<1$ & absent & absent \\
Station 8 & 10 & $<1$ & $<1$ & absent & absent \\
Station 9 & 280 & 90 & 02 & absent & absent \\
Station 10 & 120 & 98 & $<1$ & absent & absent \\
\hline
\end{tabular}

\section{DISCUSSION}

La température des eaux de la lagune Aghien a une moyenne de $32,3{ }^{\circ} \mathrm{C}$. Elle confirme un trait physique bien connu des eaux des lagunes ivoiriennes qui atteignent leurs valeurs maximales ( 30 à $32{ }^{\circ} \mathrm{C}$ ) en saison sèche (Jean-René et Daniel, 1994 ; Séraphin et al., 2008). Jean-René et Daniel (1994) expliquent que les variations spatiales de la température des eaux ont pour origine les milieux frontaliers. En effet, la profondeur moyenne $(4 \mathrm{~m})$ de la lagune, le brassage des eaux dû aux vents, aux activités de pêche, de navigation sur le plan d'eau lagunaire créent un fort hydrodynamisme qui favorise le mélange des couches d'eau de surface et des couches d'eaux sous-jacentes. Ainsi, une homogénéisation de la température sur toute la colonne d'eau se créée. En outre, les rayons du soleil traverse la faible épaisseur d'eau et la réchauffe de façon homogène (Séraphin et al., 2008; Jean-René et Daniel, 1994). La température élevée en saison sèche dans la zone d'étude entrâne une évaporation intense qui pourrait expliquer l'état basique de l'eau (Karim, 2005). Les valeurs du $\mathrm{pH}$ en lagune Aghien qui varient de 6,6 à 8,8 sont du même ordre que celles mesurées dans la lagune Potou qui varient entre 6 et 9 (Alexis, 2008). Elles sont légèrement inférieures aux valeurs observées dans les eaux de la lagune de Grand-Lahou comprises entre 7,8 et 8,1 (Séraphin et al., 2008). Les travaux de Philippe (1994); Marcel (2005); Alexis (2008) ; Séraphin et al. (2008) et Yacoub et al.
(2008) ont montré que la variation du pH est étroitement liée à celle de la salinité. Les eaux d'origine marine sont basiques et celles d'origine continentale sont acides au large d'Abidjan. Les variations spatiale et temporelle du $\mathrm{pH}$ sont liées à l'importance relative des eaux de l'une ou l'autre origine. La lagune Aghien connaît une influence marine nulle. Elle est alimentée en eau douce par les rivières Bété, Djibi et Mé pourtant ses eaux sont basiques. Cette situation pourrait s'expliquer par des réactions biologiques et physico-chimiques dues à la présence des végétaux aquatiques (Lynda et al., 2011). En effet, à l'instar de toutes les lagunes, celle d'Aghien est un milieu où vivent de nombreuses espèces végétales et animales. L'activité biologique provoque des variations nycthémérales d'autant plus nettes que les eaux sont douces, donc moins tamponnées (Philippe, 1994).

Les valeurs de la salinité sont inférieures à celles mesurées dans la lagune Potou qui sont de l'ordre de 1 à 2 (Alexis, 2008). La salinité des lagunes dépend des échanges avec l'océan, des apports d'eau douce par les rivières (Philippe, 1982 et Séraphin et al., 2008). Aussi, la faible salinité des eaux de la lagune Aghien s'explique-t-elle par l'apport en eau douce des rivières Djibi, Bété et Mé.

Les concentrations en oxygène dissous dans la lagune Aghien ont une moyenne relativement faible de 4,90 mg.L $\mathrm{L}^{-1}(66,83 \%)$. Elles sont semblables à celles déterminées par 
Séraphin et al. (2008) dans la lagune de Grand-Lahou et qui varient entre 2,8 et 4,1 mg.L $L^{-1}$. Yacoub et al. (2008) ont mesuré des concentrations du même ordre, avec une valeur moyenne de 5,16 mg. $\mathrm{L}^{-1}$ dans la lagune de Fresco. La faible concentration en oxygène dissous des eaux pourrait s'expliquer par le fait que la lagune est alimentée par les rivières Bété, Djibi et Mé et elle est chargée en MES. En effet, la concentration moyenne $(14,11$ mg. $\left.\mathrm{L}^{-1}\right)$ relativement importante des MES qui entraîne une faible transparence $(0,60$ à 1,50 $\mathrm{m})$ réduisant la pénétration de la lumière dans l'eau et la grande turbidité des rivières empêchent la photosynthèse de compenser les pertes dues à la respiration des organismes et à l'oxydation des matières organiques détritiques qu'elles charrient (Philippe, 1994). Les concentrations en MES observées pourraient être attribuées a une forte production primaire de phytoplancton et de zooplancton ou à la remise en suspension des sédiments par le brassage des vents ou encore les différentes activités anthropiques menées sur le plan d'eau lagunaire notamment la pêche, la baignade, la navigation, etc. (Philippe, 1982).

L'insuffisance d'oxygène dissous pourrait expliquer les valeurs négatives du potentiel redox dont la valeur moyenne est de - 67,7 mV. Celui-ci est associé aux réactions d'oxydoréduction et à la biodégradation des composées organiques. Lorsque l'oxygène se fait plus rare, la décomposition de la matière organique soluble devient très lente et le potentiel redox devient négatif montrant ainsi que le milieu est réducteur (Isabelle, 1999).

La $\mathrm{DBO}_{5}$ des eaux de la lagune Aghien a des valeurs comprises entre 10,95 et 26,63 mg. $\mathrm{L}^{-1}$, avec une moyenne de $17,46 \mathrm{mg} . \mathrm{L}^{-1}$ supérieure à la norme française (JODRF, 2007) (< 3 mg. $\left.\mathrm{L}^{-1}\right)$ relative à la qualité des eaux destinées à la consommation humaine. Elle est très élevée suggérant ainsi que les eaux de la lagune contiennent une quantité importante de matières organiques biodégradables. La pollution organique a plusieurs origines notamment domestique et agricole et elle est aussi le résultat de la décomposition d'animaux ou de végétaux morts (Jean-Pierre et Daniel, 1994; Daouda et al., 2011). La lagune Aghien est utilisée par les riverains pour la lessive, la baignade, la vaisselle. Ils y déposent, les restes d'aliments et les résidus de broyage de manioc perceptibles au village d'Akandjé (station 2) où existent une broyeuse de manioc et une décharge sauvage au bord de la lagune. Plusieurs fermes d'élevage existent dans la zone d'Aghien. Une partie des déjections des animaux est utilisée comme engrais dans les plantations et le reste est rejeté dans la nature. Les eaux de ruissellement et les vents entraînent ces déjections directement dans la lagune ou dans les cours d'eau qui l'alimentent. L'insuffisance de l'oxygène dissous lors des réactions conduisant à la biodégradation de la matière organique pourrait expliquer la quantité élevée $(23,04$ mg. $\left.\mathrm{L}^{-1}\right)$ des ions ammoniums par rapport à la norme JODRF (2007) qui est de 0,05 mg.L $\mathrm{L}^{-1}$. Les valeurs moyennes de $\mathrm{NO}_{3}{ }^{-}\left(4,26 \mathrm{mg} . \mathrm{L}^{-1}\right)$, $\mathrm{NO}_{2}{ }^{-}\left(0,004 \mathrm{mg} . \mathrm{L}^{-} 1\right)$ et $\mathrm{PO}_{4}{ }^{3-}\left(0,058 \mathrm{mg} . \mathrm{L}^{-} 1\right)$ des eaux de la lagune Aghien sont faibles par rapport aux normes OMS (2004) qui sont 50 mg. $\mathrm{L}^{-1} ; \quad 0,1 \quad \mathrm{mg} . \mathrm{L}^{-1} \quad$ et $\quad 0,2 \quad \mathrm{mg} . \mathrm{L}^{-1}$ respectivement. De faibles valeurs de ces nutriments ont également été observées dans les eaux de la lagune de Grand-Lahou en saison sèche par Séraphin et al. (2008). Les valeurs déterminées en $\mathrm{NO}_{3}{ }^{-}, \mathrm{NO}_{2}^{-}$et $\mathrm{PO}_{4}{ }^{3-}$ par ces auteurs varient respectivement entre 0,01 et $0,22 \mathrm{mg} . \mathrm{L}^{-1}, 0,013$ et $0,033 \mathrm{mg} . \mathrm{L}^{-1}$, 0,10 et $0,24 \mathrm{mg} . \mathrm{L}^{-1}$. Selon Philippe et Lionel (1985), les concentrations des eaux de surface en nutriments varient régulièrement sous l'influence des milieux frontières. La lagune Aghien est alimentée par les rivières Bété, 
Djibi et Mé. En saison sèche, leur influence est réduite et à cette période, on assiste à une prolifération des algues représentant une source de consommation des sels nutritifs.

Les populations riveraines déposent directement ou indirectement les fèces dans les eaux de la lagune. L'analyse bactériologique met en évidence la présence de forts taux de coliformes fécaux et totaux dans la lagune. Les streptocoques fécaux sont rares dans les eaux, les salmonelles et les vibrions y sont absents. Selon l'OMS (1992), les eaux destinées à la consommation humaine ne doivent renfermer aucun coliforme fécal. Avec des taux de coliformes supérieurs à 100 coliformes/100 ml, les eaux de la lagune Aghien sont polluées et inutilisables pour les usages domestiques. Les coliformes thermotolérants, les entérocoques et les bactéries anaérobies sulfito-réductrices sont des indicateurs de contamination fécale (Séraphin et al., 2009) et le grand nombre de ces types de bactéries pourrait indiquer la présence d'autres types de bactéries potentiellement dangereux pour la santé de l'homme.

\section{Conclusion}

Les eaux de la lagune Aghien ont une salinité moyenne de 0,03 . Elles possèdent une quantité importante de matières organiques biodégradables traduite par une $\mathrm{DBO}_{5}$ moyenne de 17,46 mg/l. Les concentrations en ion ammonium de ces eaux sont très élevées, avec une moyenne de 23,04 mg/l mais les concentrations moyennes en ions nitrates, nitrites et orthophosphates y sont très faibles, avec des valeurs respectives de $4,25 \mathrm{mg} / \mathrm{l}$, $0,004 \mathrm{mg} / \mathrm{l}$ et $0,05 \mathrm{mg} / \mathrm{l}$. La comparaison des teneurs en microorganismes par rapport aux normes de l'OMS pour les eaux de boisson montre que les eaux de la lagune Aghien sont polluées avec une présence massive de coliformes totaux et fécaux par endroits. Ces eaux ont besoin d'être protégées et gérées de façon rationnelle pour non seulement la préservation de la ressource en eau mais également pour la protection de l'écosystème aquatique et ses potentialités biologiques.

\section{REMERCIEMENTS}

Nous remercions le Docteur Aké Assi Yolande, Chef du Laboratoire Central pour l'Hygiène Alimentaire et l'Agro-industrie (LCHAI), qui a mis à notre disposition le laboratoire au sein duquel toutes les analyses ont été effectuées.

\section{REFERENCES}

Alexis NY. 2008. Analyse morphologique, sédimentologique et environnement de dépôts des sédiments superficiels des lagunes Adjin et Potou (Zone littorale de la Côte d'Ivoire). Thèse de Doctorat de l'Université de Cocody, p. 145.

Claude D, Ibrahima D, Yves S, M'bé A, Zamblé ZB, Jean-Pierre T, Bertin DY, Gilbert K, Jean-Claude C, Alain D. 1992. Carte géologique de la Côte d'Ivoire à $1 / 200000,1^{\text {ère }}$ Edn, Feuille GRANDBASSAM, Direction de la Géologie, Abidjan, Côte d'Ivoire, 1992.

Daouda M, Véronique D, James B, Waris C, Benjamin Y, Baba G, Michel B. 2011. Caractérisation d'un Système Lagunaire en Zone Tropicale: Cas du lac Nokoué (Bénin). European Journal of Scientific Research, 56(4): 516-528.

Ernest AK, Nagnin S, Gbombélé S, Théophile L, Solange OM, Pacôme ZS. 2008. Groundwater Pollution in Africans Biggest Towns: Case of the Town of Abidjan (Côte d'Ivoire). European Journal of Scientific Research, 20(2): 302-316.

Isabelle M .1999. Infiltration des eaux de ruissellement pluvial et transfert de polluants associés dans le sol urbain - 
Vers une approche globale et pluridisciplinaire. Thèse de Doctorat, Institut National des Sciences Appliquées de Lyon, p. 207.

Daniel G. 1994. Géologie et sédimentologie. In Environnement et Ressources Aquatiques de Côte d'Ivoire : Les Milieux Lagunaires (Tome II). Éditions de l'ORSTOM : Paris; 35-57.

Jean-René D, Daniel G. 1994. Hydroclimat et Hydrochimie. In Environnement et Ressources Aquatiques de Côte d'Ivoire: Les Milieux Lagunaires (Tome II). Éditions de l'ORSTOM : Paris; 59-90.

Journal officiel de la République Française. 2007. Limites et références de qualité des eaux destinées à la consommation humaine, à l'exclusion des eaux conditionnées. Ministère de la Santé et des Solidarités. Arrêté du 11 janvier 2007 relatif aux limites et références de qualité des eaux brutes et des eaux destinées à la consommation humaine mentionnées aux articles R. 1321-2, R. 1321-3, R. 1321-7 et R. 1321-38 du code de la santé publique, p. 9 .

Juliette DV, Marianne S, Catherine Y. 2005. Qualité physico-chimique et chimique des eaux de surface: cadre général. Institut Bruxellois pour la Gestion de l'Environnement. Observatoire des Données de l'Environnement, p. 16.

Karim BM. 2005. Etude géochimique de la lagune de Nador (Maroc oriental): Impacts des facteurs anthropiques. Thèse de Doctorat, Université Mohamed VAgdal, p.215.

Lynda E, Tchirioua E, Jules NK, Toussaint D. 2011. Variations temporelles des paramètres physicochimiques et biotiques de deux écosystèmes aquatiques de la lagune Ebrié. European Journal of Scientific Research, 58(3): 414-422.
Marcel AK. 2005. Hydrochimie et qualité des eaux de deux lagunes tropicales de Côte d'Ivoire (Ebrié, Grand-Lahou). Thèse de Doctorat, Université de Cocody, Abidjan, p. 242.

Ministère des Eaux et Forêts (MEF). 2003. Gestion intégrée des ressources en eau en Côte d'Ivoire. Bilan et perspectives. Ministère des Eaux et Forêts, Côte d'Ivoire, p. 59.

Murphy J, Ryley J. 1962. A modified single solution method for the determination of phosphate in natural waters. Anal. Chim. Acta, 27: 31-36.

Norme française. 1975. Méthodes de dosage de l'azote ammoniacal. NF $\mathrm{T}$ 90-015; 332-337.

Norme Française. 1978. Méthodes de dosage des matières en suspension. NF T 90-105, 273-277.

Norme Française. 1987. Essais des eauxDosage des nitrites-Méthode par spectrométrie moléculaire. NF T 90-013; 314-319.

OMS. 1992. Directives de la qualité pour l'eau de boisson. OMS, $\mathrm{N}^{\circ} 1,192$.

OMS. 2004. Guidelines for drinking water quality, volume 1, recommendations. First addendum to $3^{\text {rd }}$ edn. World Health Organization (WHO), Geneve, Suisse; p.595.

Patrice JJ, Jean KK, Bachir SM, Hélène KB, Solange OY. 2006. Contamination of the Abidjan aquifer by sewage: An assessment of extent and strategies for protection. In Groundwater Pollution in Africa, Yongxin X, Brent U (eds). Taylor \& Francis/Balkema: Great-Britain; 293302.

Philippe D. 1982. Les frontières naturelles et humaines du système lagunaire Ebrié. Incidences sur l'hydroclimat. Hydrobiologia, 94: 105-120. 
Philippe D. 1984. Production primaire d'une lagune tropicale (Ebrié, Côte-d'Ivoire). Facteurs naturels et anthropiques. Thèse, Univ. Pierre et Marie-Curie, Paris, p. 164.

Philippe D. 1994. Du biotope à la biocénose. In Environnement et Ressources Aquatiques de Côte d'Ivoire : Les Milieux Lagunaires (Tome II). Éditions de l'ORSTOM : Paris; 93-108.

Philippe D, Lionel L. 1985. Le régime nutritif de la lagune tropicale Ebrié (Côte d'Ivoire). Océanogr. Trop., 20(1): 41-69.

Robert A, Daniel G, Jean-Pascal T. 1989. Cycle hydrologique annuel d'une baie lagunaire eutrophe : la baie de Biétri (lagune Ebrié, Côte d'Ivoire). Rev. Hydrobiol. Trop., 22(4): 263-273.

Séraphin KK, Marcel AK, Antoinette AA, Dago G. 2009. Spatial and temporal variation of fecal contamination indicators in Grand-Lahou lagoon, Côte d'Ivoire. Journal of Applied Biosciences, 23: 1422-1435.

Séraphin KK, Marcel AK, Antoinette AA, Dongui BK, Dago G. 2008. Variations saisonnières des paramètres abiotiques des eaux d'une lagune tropicale: la lagune de Grand-Lahou, Côte d'Ivoire. European Journal of Scientific Research, 21(3): 376-393.

Yacoub I, Marcel AK, Dongui BK, Jean B. 2008. Caractéristiques physico-chimiques d'une lagune côtière tropicale : lagune de Fresco (Côte d'Ivoire). Afrique Science, 4(3): 368-393. 